\title{
Continuous Thermodynamic Integration in Field-Theoretic Simulations of Structured Polymers
}

\author{
Russell K. W. Spencer* and Mark W. Matsen \\ Department of Chemical Engineering, Department of Physics 63 Astronomy, \\ and Waterloo Institute for Nanotechnology, University of Waterloo, Waterloo, Ontario, Canada \\ Bart Vorselaars \\ School of Mathematics and Physics, University of Lincoln, \\ Brayford Pool, Lincoln, LN6 7TS, United Kingdom
}

(Dated: July 27, 2017)

\begin{abstract}
This work explores the use of continuous thermodynamic integration (TI) in field-theoretic simulations of a symmetric diblock copolymer melt. Free energies of the lamellar and disorder phases are evaluated by thermodynamic integration from a reference state (an Einstein crystal, $\lambda=0$ ) to a diblock copolymer $(\lambda=1)$. This is followed by integration over the interaction parameter, $\chi_{b}$, to locate the order-disorder transition (ODT). We then examine the equilibrium lamellar spacing and free energy cost of stretching and compressing lamellae. The ODT, lamellar spacing, and compression modulus are consistent with previous calculations, though found faster and more precisely. The above quantities do not depend on simulation box size, suggesting that finite-size effects are small and simulating 2 lamellar periods is sufficient to accurately evaluate bulk behavior. Furthermore, the statistical uncertainty in the ODT increases quickly with system size, suggesting that small systems may lead to more precise results.
\end{abstract}

\section{INTRODUCTION}

Field-theoretic simulations (FTS) offer an efficient way of simulating high molecular weight polymers and are able to capture fluctuation effects, which are absent from mean-field theories. Although FTS do not directly evaluate free energies, indirect methods, such as thermodynamic integration (TI), can be used to find free energy differences. This work builds on promising TI techniques in FTS, by adapting a continuous TI method in order to improve the speed, accuracy and precision of such methods.

Calculating free energies using TI allows one to locate first-order phase transitions without having to simulate structural transformations between phases. This is a major improvement over other simulation methods, such as metastability interval calculations, ${ }^{1}$ particularly for strongly first-order transitions, which can take prohibitively long to occur. The use of TI in field-based polymer simulations was pioneered by Lennon and coworkers, who used it to calculate the phase diagram for a diblock copolymer melt. ${ }^{2}$ Their method was adapted from the particle-based method of Frenkel and Ladd, ${ }^{3}$ where free energies are computed by integrating from an Einstein crystal (EC) reference state. Calculating the free energy relative to a reference state with known free energy allows for the evaluation of absolute, not merely relative, free energies. Using a reference state also facilitates the examination of order-order transitions, unlike methods that rely on external ordering fields to create closed loops in configuration space. ${ }^{4}$ The work of Lennon et al. was extended by Delaney and Fredrickson to more carefully evaluate the phase diagram, as well as the compression modulus of the lamellar phase and the behavior of homopolymers. ${ }^{5}$
In the traditional TI method, employed in the work described above, ${ }^{2,5}$ a number of simulations is conducted over the range of the integration parameter. Each simulation calculates a thermodynamic average, which gives the derivative of the free energy at that point. These averages are then used to integrate the free energy. This can take many simulations and is generally computationally expensive. It also leads to complicated considerations for evaluating and managing statistical uncertainties. One must decide how long to equilibrate simulations before collecting statistics, to reduce a systematic equilibration error; how long to collect statistics for averages, which contain a random error; and how many steps in parameter space are required for an acceptable finite step error. The random error in thermodynamic averages propagates through the integral and is quantifiable using standard techniques. The equilibration and finite-step errors are more difficult to quantify.

Here, we adopt an alternative TI scheme, involving only one simulation per integral. The integration variable is incremented slowly, throughout the simulation, and the integral is performed using instantaneous quantities, rather than their thermodynamic averages, as free energy derivatives. This continuous method is also known as adiabatic switching, slow growth, or single-configuration TI, and is well-established in particle-based simulations. ${ }^{6-9}$ In addition to its speed and simplicity, continuous TI also offers a simple way of quantifying and minimizing errors. The large number of steps, and thus small step size, renders finite-step errors negligible. Random errors are quantifiable and are typically small. ${ }^{6}$ The main source of error is systematic and is due to the system being out of equilibrium as it is dragged through parameter space. Since the sign of this error depends on the direction the system is dragged, the true value can be bracketed by 
conducting a second integral from the final state back to the initial state.

Our testing ground for continuous TI is a melt of symmetric diblock copolymers, for which we study finite-size effects in the order-disorder transition (ODT), domain spacing and compression modulus. One would expect that as the simulated region increases in size, thermodynamic quantities should monotonically approach the bulk value. The domain spacing and ODT, however, have been seen to vary non-monotonically with simulation box size. ${ }^{10}$ Subsequent work has concluded that once the simulation box is commensurate with the underlying microstructure, results are consistent between simulations of different size. ${ }^{11}$ This may not always be the case, as non-monotonic changes in the ODT with system size have been suggested in simulations, even when the box lengths can adopt the preferred domain size. ${ }^{1}$ That ODT determination was, however, not precise enough to be definitive for experimentally relevant molecular weights.

\section{THEORY}

Our work examines an incompressible melt of $n \mathrm{AB}$ diblock copolymers contained in a volume $V$. The A and B blocks are composed of $f N$ and $(1-f) N$ segments respectively. The polymers have an invariant polymerization index $\bar{N}=a^{6} \rho_{0}^{2} N$, where $a$ is the segment length and $\rho_{0}$ is the monomer density. The interaction between $\mathrm{A}$ and $\mathrm{B}$ monomers is characterized by the Flory-Huggins interaction parameter, $\chi_{b}$. In the following calculations and simulations, lengths are given in terms of $R_{0}=a \sqrt{N}$.

Statistical Mechanics. The partition function for our system,

$$
Z \sim \int \exp \left(-\frac{H\left[W_{-}, W_{+}\right]}{k_{B} T}\right) \mathcal{D} W_{-} \mathcal{D} W_{+},
$$

is written in terms of the fluctuating fields, $W_{-}(\mathbf{r})$ and $W_{+}(\mathbf{r})$ and a composite Hamiltonian,

$$
H\left[W_{-}, W_{+}\right]=\lambda H_{\mathrm{BCP}}\left[W_{-}, W_{+}\right]+(1-\lambda) H_{\mathrm{EC}}\left[W_{-}\right],
$$

which is a linear combination of block copolymer (BCP) and Einstein crystal (EC) Hamiltonians. In the BCP limit, $\lambda=1$, this model can be derived using standard techniques, ${ }^{12-14}$ resulting in the Hamiltonian

$$
\frac{H_{\mathrm{BCP}}\left[W_{-}, W_{+}\right]}{n k_{B} T}=\ln \left(\frac{V}{Q}\right)+\frac{1}{V} \int\left(\frac{W_{-}^{2}}{\chi_{b} N}-W_{+}\right) d \mathbf{r} .
$$

The fields, $W_{-}(\mathbf{r})$ and $W_{+}(\mathbf{r})$, act on the difference, $\hat{\phi}_{-}(\mathbf{r})=\hat{\phi}_{A}(\mathbf{r})-\hat{\phi}_{B}(\mathbf{r})$, and total, $\hat{\phi}_{+}(\mathbf{r})=\hat{\phi}_{A}(\mathbf{r})+\hat{\phi}_{B}(\mathbf{r})$, of the instantaneous concentrations of $\mathrm{A}$ and $\mathrm{B}$ segments. The partition function of a single polymer in the $W_{-}(\mathbf{r})$ and $W_{+}(\mathbf{r})$ fields,

$$
Q\left[W_{-}, W_{+}\right]=\int q(\mathbf{r}, 1) d \mathbf{r}
$$

is written in terms of a partial partition function, $q(\mathbf{r}, s)$, which satisfies

$$
\frac{d q(\mathbf{r}, s)}{d s}=\left[\frac{R_{0}^{2}}{6} \nabla^{2}-\frac{1}{2}\left(W_{+} \pm W_{-}\right)\right] q(\mathbf{r}, s),
$$

with ' + ' for $0<s<f$ and ' - ' for $f<s<1$, where $s$ is the position along the chain. The diffusion equation is solved using a fourth-order pseudospectral method ${ }^{15}$ with initial condition $q(\mathbf{r}, 0)=1$.

The EC model considers $W_{-}(\mathbf{r})$ at each point in our simulation to be an independent oscillator, varying about some reference value, $W_{0}(\mathbf{r})$. The corresponding Hamiltonian is found by integrating the energies of all oscillators in the system

$$
\frac{H_{\mathrm{EC}}\left[W_{-}\right]}{n k_{B} T}=\frac{\alpha}{2 V} \int\left(W_{-}(\mathbf{r})-W_{0}(\mathbf{r})\right)^{2} d \mathbf{r}
$$

where $\alpha$ is the spring constant for the oscillators. Throughout this work, we use the EC as a reference state and set its free energy to zero. The free energy of the EC is not uniquely defined in the continuum limit, however, the field $W_{-}(\mathbf{r})$, and thus the Einstein crystal, will later be discretized onto a lattice with $M$ points. When integrating along $\lambda$, we want a smooth transformation between EC and BCP, with no phase transitions, and thus choose a $W_{0}(\mathbf{r})$ which reflects the symmetry of each phase of interest. Since the free energy per unit volume of the EC only depends on $\alpha$, it is convenient to use the same $\alpha$, and thereby have the same reference free energy, when comparing the disorder and lamellar phases.

Discretization and the Ultraviolet Divergence. For calculation purposes, the fields $W_{-}(\mathbf{r})$ and $W_{+}(\mathbf{r})$, are defined on discrete grids, with grid spacings $\Delta_{x}, \Delta_{y}$, and $\Delta_{z}$, and side lengths $L_{x}, L_{y}$ and $L_{z}$. We typically use uniform, cubic grids with spacing $\Delta$ and length $L$. When it deviates from cubic, the box is stretched or compressed along the $x$-direction, which is normal to the lamellae and denoted by $\perp$. The volume, $V=L_{x} L_{y} L_{z}$, of the system is conserved by setting the other lengths to $L_{y}=$ $L_{z}=L_{\|}=\sqrt{V / L_{\perp}}$. Lengths are altered by changing grid spacings, $\Delta_{\perp}=\Delta_{x}$ and $\Delta_{\|}=\Delta_{y}=\Delta_{z}$, while maintaining the number of points along each direction.

One might expect that as $\Delta$ decreases, simulations would become more accurate and the free energy would converge. On the contrary, decreasing $\Delta$ leads to an increasing contribution to the free energy, which acts to disorder the melt. This ultraviolet (UV) divergence is explored more thoroughly in previous work. ${ }^{1,16}$ In order to compare with experiments, or even similar simulations with a different $\Delta$, the diverging contribution to the free energy is absorbed into the monomer interaction energy, thereby renormalizing the bare interaction parameter, $\chi_{b}$, to obtain $\chi_{e}$. We calculate the effective interaction parameter using the method of Olvera de la $\mathrm{Cruz}^{17}$ to obtain

$$
\frac{\chi_{e}}{\chi_{b}}=1-\frac{12 R_{0}}{\pi^{3} \sqrt{\bar{N}}} \int_{0}^{\Lambda_{\|}} \int_{0}^{\Lambda_{\|}} \int_{0}^{\Lambda_{\perp}} \frac{d k_{x} d k_{y} d k_{z}}{k_{x}^{2}+k_{y}^{2}+k_{z}^{2}},
$$


where $\Lambda_{\gamma}=\pi / \Delta_{\gamma}$ is the wave vector cutoff in the $\gamma$ direction.

Simulation Method. Our Monte Carlo fieldtheoretic simulations (MC-FTS) are based on techniques more fully described in previous work. ${ }^{18,19}$ Solving Equation (1) using Monte Carlo (MC) simulations becomes complicated because $W_{+}(\mathbf{r})$ in Equations (1) and (2) takes on imaginary values, making standard simulation techniques impossible. We therefore employ the saddlepoint approximation to $W_{+}(\mathbf{r})$, to obtain $w_{+}(\mathbf{r})$, which enforces incompressibility in a mean-field way,

$$
\phi_{+}(\mathbf{r})=\frac{V}{Q} \int_{0}^{1} q(\mathbf{r}, s) q^{\dagger}(\mathbf{r}, s) d s=1,
$$

where $q^{\dagger}(\mathbf{r}, s)$ is defined similarly to $q(\mathbf{r}, s)$, and is found by propagating from the other end of the molecule. For each $W_{-}(\mathbf{r})$, we adjust $w_{+}(\mathbf{r})$ iteratively using Anderson mixing $^{20}$ until

$$
\left[\frac{1}{V} \int\left(\phi_{+}(\mathbf{r})-1\right)^{2} d \mathbf{r}\right]^{1 / 2}<\varepsilon .
$$

A tolerance of $\varepsilon=10^{-4}$ is sufficient for our calculations. Employing the saddle-point approximation to $W_{+}(\mathbf{r})$ reduces Equation (1) to

$$
Z \sim \int \exp \left(-\frac{H\left[W_{-}, w_{+}\right]}{k_{B} T}\right) \mathcal{D} W_{-},
$$

where the integral is only over $W_{-}(\mathbf{r})$. The fields, $W_{-}(\mathbf{r})$ and $w_{+}(\mathbf{r})$, are both real-valued, and thus the Hamiltonian is also real. As a result, we are free to use any standard MC technique.

Each simulation step involves making a small change to the system, usually by changing $W_{-}(\mathbf{r})$, followed by calculating the Hamiltonian, $H\left[W_{-}, w_{+}\right]$, and using standard Metropolis MC criteria to accept or reject these changes. We alternate between a real-space move, where the change in $W_{-}(\mathbf{r})$ at each grid point is selected from a uniform distribution, and a Fourier-space move, where the change in $W_{-}(\mathbf{k})$ at each wavevector is selected from a uniform distribution weighted by the FredricksonHelfand structure function, $S_{\mathrm{FH}}(k) .{ }^{21}$ The amplitude of each move type is adjusted during an equilibration period, to achieve an acceptance probability of $\sim 40 \%$. Some of our simulations for the lamellar phase also employ a box move, where the box dimensions are changed, while holding the volume, $V=L_{\perp} L_{\|}^{2}$, constant.

Thermodynamic Integration. We use thermodynamic integration to calculate the relative free energy between states, by integrating along a path in parameter space that connects those states. When locating the order-disorder transition (ODT) we start at $\lambda=0$, where both the disorder and lamellar phases have the same free energy (that of the EC), then conduct a simulation to integrate to $\lambda=1$ (pure BCP). We then integrate along $\chi_{b}$, which gives us $F$ vs. $\chi_{b} N$ for both phases, and thus the ODT.
Each integral is conducted along an integrand $X=\lambda$, $\chi_{b}$ or $L_{\perp}$, from $X_{s}$ to $X_{f}$, using $\mathcal{M}$ steps. At each step, a MC move is performed, $X$ is incremented by $\Delta X=\left(X_{f}-X_{s}\right) / \mathcal{M}$ and the free energy is incremented by $\Delta X \times d H / d X$. There are two sources of error: systematic errors due to the system being out of equilibrium, as it tends to be closer to the initial than final state; and random errors, which are much smaller than the systematic errors. Both errors can be reduced by increasing $\mathcal{M}$. If we evaluate two integrals, one from $X_{s}$ to $X_{f}$ and another back to $X_{s}$, we can quantify the systematic error. The systematic shift, which behaves like a dissipative drag force, causes thermodynamic integration to overestimate the free energy difference. ${ }^{22}$ The first integral overestimates the free energy difference $F_{f}-F_{s}$, whereas the second integral overestimates $F_{s}-F_{f}$, and thus underestimates $F_{f}-F_{s}$. The two estimates are therefore upper and lower bounds on the true free energy difference. Although the system is only in true equilibrium in the limit $\mathcal{M} \rightarrow \infty$, a sufficiently large $\mathcal{M}$ reduces the aforementioned systematic error to an acceptable level. We estimate how large $\mathcal{M}$ must be using short trials on small systems and integrals over small ranges of $X$.

We wish to have similar distributions of $W_{-}(\mathbf{r})$ in the BCP and EC systems, so that the system changes little throughout the $\lambda$ integral. This is achieved by setting $W_{0}(\mathbf{r})$ to the most likely $W_{-}(\mathbf{r})$ field - that found from self-consistent field theory (SCFT), and choosing an $\alpha$ which allows the fluctuations in the Einstein crystal to mimic that of a block copolymer. The ideal $\alpha$ can be determined in a number of ways: finding the standard deviation of $W_{-}(\mathbf{r})$ in a MC-FTS simulation; analytically determining the second derivative, $\mathcal{D}^{2} H_{\mathrm{BCP}} / \mathcal{D} W_{-}(\mathbf{r})^{2}$; or semi-analytically, by taking the above functional derivative in a SCFT calculation. The results from all three methods are close and do not depend strongly on position or phase. We use $\alpha=2 / \chi_{b} N$. The procedure for locating the ODT is simpler if the reference free energies for both phases are the same, making it more convenient to use the same $\alpha$ for both ECs. Since $\alpha$ depends on $\chi_{b} N$ and we examine two values of $\chi_{b} N$, we use an $\alpha$ corresponding to their midpoint.

Such care need not be taken in choosing the ideal values of $W_{0}(\mathbf{r})$ and $\alpha$, as the final results are not sensitive to these choices. The reference field, $W_{0}(\mathbf{r})$, may, for example, be chosen from any equilibrated BCP simulation. These different choices of $W_{0}(\mathbf{r})$ can yield different 'paths,' $F$ vs. $\lambda$, however, the total free energy difference is the same within the (small) random error. Different choices of $\alpha$ merely lead to a change in the EC free energy, and thus a constant shift in the free energy difference between EC and BCP. The only inappropriate choices of $W_{0}(\mathbf{r})$ and $\alpha$ appear to be those where a phase transition exists in the range $0 \leq \lambda \leq 1$. This is because TI is only applicable when the free energy is continuous and differentiable. Examples of such inappropriate choices are $W_{0}(\mathbf{r})$ which does not have the symmetry of the phase of interest, and $\alpha$ so small that random variation in $W_{-}(\mathbf{r})$ 
dominates and the EC loses the structure of the phase.

Free Energy Derivatives. Thermodynamic integration involves calculating derivatives of the free energy with respect to system parameters, and stepping along these parameters, integrating the free energy. We perform three integrals: over $\lambda$, from the $\mathrm{EC}$ to the $\mathrm{BCP}$; over $\chi_{b}$; and over domain spacing. Each derivative corresponds to an ensemble average of the derivative of the Hamiltonian with respect to the parameter of interest,

$$
\frac{d F}{d X}=-\frac{k_{B} T}{Z} \frac{d Z}{d X}=\left\langle\frac{d H}{d X}\right\rangle .
$$

For integrating over $\lambda$, the derivative is given by

$$
\frac{d F}{d \lambda}=\left\langle H_{\mathrm{BCP}}-H_{\mathrm{EC}}\right\rangle
$$

We also need to integrate over $\chi_{b}$, with $\lambda=1$. The resulting derivative is

$$
\frac{d F}{d \chi_{b}}=-\frac{\int\left\langle W_{-}^{2}\right\rangle d \mathbf{r}}{V \chi_{b}^{2} N}
$$

Finally, we wish to integrate over domain spacing, $D=$ $L_{\perp} / h$, where $h$ is the number of periods in the simulation box. This integration requires

$$
\frac{d F}{d L_{\perp}}=\left\langle\frac{d H_{\mathrm{BCP}}}{d L_{\perp}}\right\rangle .
$$

If $\chi_{b}$ remains constant throughout the simulation, then only the $\ln Q$ term in Equation (3) contributes to the derivative, Equation (14). Changing $L_{\perp}$ by altering the grid spacings, however, can have a significant effect on the free energy, through the UV divergence. Keeping $\chi_{e}$ constant, by changing $\chi_{b}$, introduces a second term to $d H_{\mathrm{BCP}} / d L_{\perp}$, leading to

$$
\frac{1}{n k_{B} T} \frac{d H_{\mathrm{BCP}}}{d L_{\perp}}=-\frac{1}{Q} \frac{d Q}{d L_{\perp}}-\frac{\int W_{-}^{2} d \mathbf{r}}{\chi_{b}^{2} N V}\left(\frac{\partial \chi_{b}}{\partial L_{\perp}}\right)_{\chi_{e}, V} .
$$

The first term

$$
\frac{d Q}{d L_{\perp}}=\frac{R_{0}^{2}}{3(2 \pi)^{3} L_{\perp}} \int q(\mathbf{k}, s) k_{x}^{2} q^{\dagger}(-\mathbf{k}, s) d \mathbf{k} d s,
$$

was derived previously. ${ }^{23}$ Recall that we keep the volume constant when changing $L_{\perp}=L_{x}$ by changing the other box dimensions, such that $L_{\|}=L_{y}=L_{z}=\sqrt{V / L_{\perp}}$. From symmetry, the $d Q / d L_{\|}$terms average to zero in the lamellar phase and can be ignored. The second term in Equation (15), is found by differentiating Equation $(7)$, at fixed $\chi_{b}$ and $V$,

$$
\begin{aligned}
\left(\frac{\partial \chi_{e}}{\partial L_{\perp}}\right)_{\chi_{b}, V} & =\frac{12 R_{0} \chi_{b}}{\pi^{2} L_{\perp} \sqrt{\bar{N}}}\left[\frac{1}{\Delta_{\perp}} \int_{0}^{\Lambda_{\|}} \int_{0}^{\Lambda_{\|}} \frac{d k_{y} d k_{z}}{\Lambda_{\perp}^{2}+k_{y}^{2}+k_{z}^{2}}\right. \\
& \left.-\frac{1}{\Delta_{\|}} \int_{0}^{\Lambda_{\|}} \int_{0}^{\Lambda_{\perp}} \frac{d k_{x} d k_{y}}{k_{x}^{2}+k_{y}^{2}+\Lambda_{\|}^{2}}\right]
\end{aligned}
$$

and using

$$
\left(\frac{\partial \chi_{b}}{\partial L_{\perp}}\right)_{\chi_{e}, V}=\frac{\chi_{b}}{\chi_{e}}\left(\frac{\partial \chi_{e}}{\partial L_{\perp}}\right)_{\chi_{b}, V}
$$

Although the true derivatives are given by thermodynamic averages, recall that continuous thermodynamic integration substitutes instantaneous values of $d H / d X$.

\section{RESULTS}

This work examines the equilibrium properties of a melt of symmetric diblock copolymers, $f=1 / 2$, with invariant polymerization index $\bar{N}=10^{4}$. The melts are contained in cubic or cuboidal boxes, represented on discrete grids with periodic boundary conditions. Box lengths are chosen in order to fit $h$ lamellar periods, with lamellae parallel to a box side. Grid spacings are chosen such that there are 8 points per lamellar period, for a total of $M=(8 h)^{3}$ points.

Our first task is to determine the order-disorder transition (ODT). We start by calculating the free energy of a block copolymer melt (BCP) in the disordered phase (DIS) at $\chi_{b} N=13$ and the lamellar phase (LAM) at $\chi_{b} N=17$. The DIS and LAM free energies are found relative to an Einstein crystal (EC) of $M$ oscillators with spring constant $\alpha$. This is accomplished by thermodynamically integrating from $\lambda=0$ to 1 . Examples of these integrals are shown in Figure 1. Integrating from the EC to the BCP and back to the EC gives an estimate of the error in the free energy. This error is found to be small, and is illustrated in the inset, which emphasizes the first $1 \%$ of the range of $\lambda$. The error increases with system size and is slightly larger for LAM than DIS.

Once we have obtained the free energy of DIS and LAM at specific (different) values of $\chi_{b} N$, it is time to integrate along $\chi_{b} N$ and find the ODT. Results of these integrals are shown in Figure 2. Integrals (forward) from DIS start at $\chi_{b} N=13$ and from LAM start at $\chi_{b} N=17$. When integrating back, we start soon after the ODT found in the forward calculation. Only the ranges of $\chi_{b} N$ close to the ODT are shown. The main source of error in the free energy is a systematic error associated with being dragged through the range of $\lambda$. For the most part, this error appears to affect DIS and LAM the same - there is an overall shift in the free energies. There is, however, a small difference in how it affects DIS and LAM, which causes a difference in the ODT. We expect the shift in the ODT to go one direction (higher or lower $\left.\chi_{b} N\right)$ when integrating 'forward' and the other direction (lower or higher $\chi_{b} N$ ) on integrating 'back.' The true ODT is probably close to the middle of the bounds.

We wish to determine the ODT at the equilibrium LAM spacing. One way to do this is to allow the box size to vary during the simulation. It is simpler, however, to find the equilibrium spacing and fix the box dimensions. We therefore find the ODT at the spacing determined 

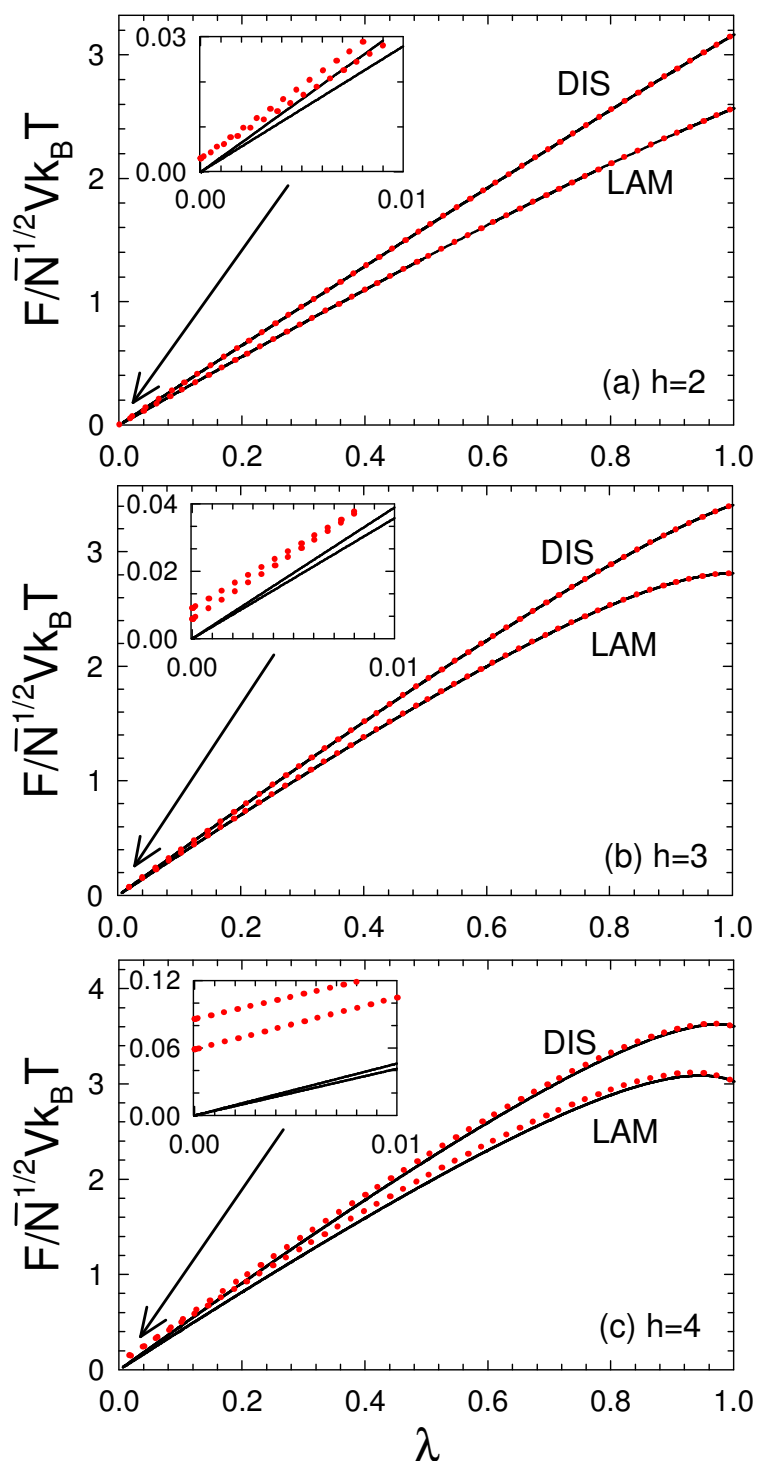

FIG. 1. Free energy of DIS $\left(\chi_{b} N=13\right)$ and LAM $\left(\chi_{b} N=\right.$ 17 ) in a system with a composite Hamiltonian, Equation (2), relative to that of an Einstein crystal. The results of forward $(\lambda=0$ to 1$)$ integrals are shown in black lines, and back $(\lambda=1$ to 0$)$ in red dots. Free energies are calculated for systems large enough to contain (a) 2, (b) 3 and (c) 4 periods. The inset emphasizes the data close to $\lambda=0$ and thus the difference between free energies evaluated by integrating in forward and back directions. Integrals were done using $10^{7}$ steps.

by Vorselaars et al. ${ }^{1}$ then find the LAM spacing using the box move, followed by a second determination of the ODT. In each case the difference in ODT and in LAM spacing between the first and second determination was small.

Once we have found the ODT, we can compare the results for 2, 3 and 4 periods, shown in Figure 3. There is no significant difference in the ODT for systems of different size, suggesting that finite-size effects on the ODT are small. The difference between upper and lower
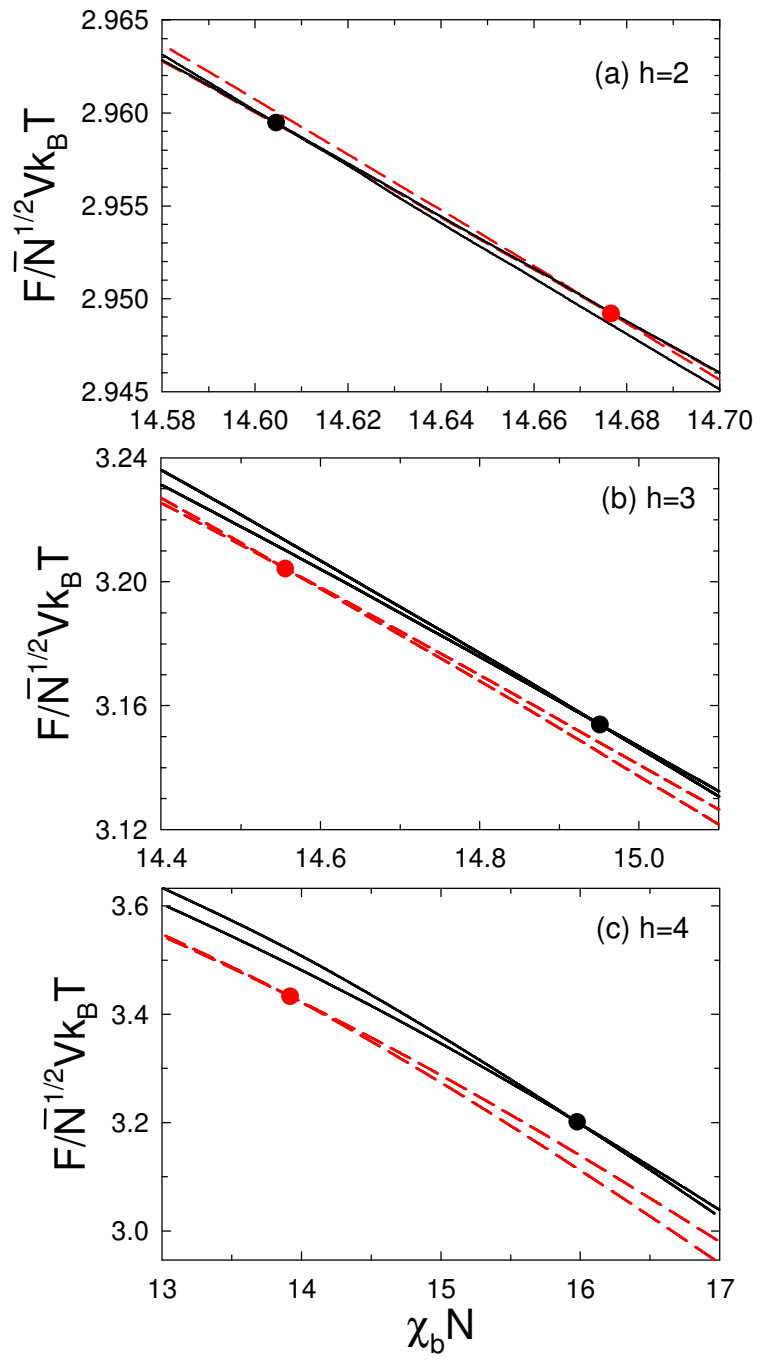

FIG. 2. Free energy of DIS and LAM, calculated by integrating forward (black solid) and back (red dashes) along $\chi_{b} N$, subsequent to forward and back $\lambda$ integrals respectively. Data are shown for systems large enough to contain (a) 2, (b) 3 and (c) 4 periods. Points indicate the ODT. ODTs determined by forward and back integration are taken as upper and lower bounds. Each integral was done using $10^{6}$ steps.

bounds for the ODT, however, increases drastically as the number of periods is increased. This is because large systems take longer to equilibrate, effectively increasing the work required to drag the system through parameter space and thereby increasing the systematic error. Our results are consistent with those found by Vorselaars et al. who bracketed the ODT by finding a range of $\chi_{b} N$ over which DIS and LAM were metastable throughout long simulations. ${ }^{1}$ The thermodynamic integration approach was able to go to a larger system size than the metastability interval approach. Where Vorselaars et al. ${ }^{1}$ were able to go to $h=4$, their results suggested non-monotonic changes in the ODT. We do not see this effect, however, the large error bars for $h=4$ leave open 


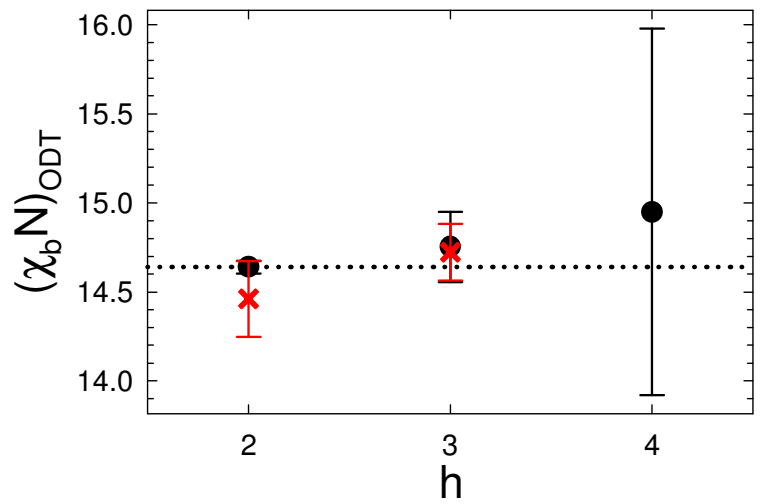

FIG. 3. ODT determined by thermodynamic integration (black dots) and a metastability intervals ${ }^{1}$ (red crosses) for $h=2,3$ and 4 periods. In the TI data, the range is calculated as in Figure 2 and the point indicates the midpoint of that range. The horizontal dotted line is through the $h=2$ ODT and is a guide to the eye.

the possibility.

The integrals illustrated in Figures 1 and 2 were done using the same numbers of steps, and thus the same rates, $\Delta \lambda=10^{-7} /$ step and $\Delta \chi_{b} N=4 \times 10^{-6} /$ step. As the system size increases, the relaxation time increases, thereby increasing the error in the free energy and thus the ODT. This means that as system size increases, not only do simulations become slow (since computational time for each MC step scales as $V \ln V$ ) but the error also increases rapidly, meaning that simulations need to be done even slower to reduce this error. Fortunately, we see no sign of finite size effects and small systems may be sufficient to determine the ODT.

We now turn our attention to the equilibrium lamellar spacing and the free energy cost of deviating therefrom. To evaluate these, we integrate along the lamellar spacing, typically starting from the equilibrium spacing determined using the box move. Along with thermodynamic integration, we can find the (relative) free energy of stretching and compressing lamellae by employing the box move in a Monte Carlo simulation. The box move technique has been employed to find the equilibrium lamellar spacing and compression modulus. ${ }^{1} \mathrm{~A}$ comparison is shown in Figure 4. The two approaches yield consistent results for the free energy, and thus quantities derived therefrom, such as the equilibrium spacing and compression modulus. To find $F$ in the range of Figure 4, TI requires an order of magnitude fewer steps than the box move. The range of $D$ that TI considers scales linearly with number of steps, whereas, for the box move, the number of steps required to sample a range of $D$ increases exponentially. This is because the probability of lamellae adopting a spacing, $D$, in the box move scales as a Boltzmann weight of the free energy cost of adopting that $D$.

The next step is to reevaluate the data in Figure 4 at different system sizes, in order to look for finite-size

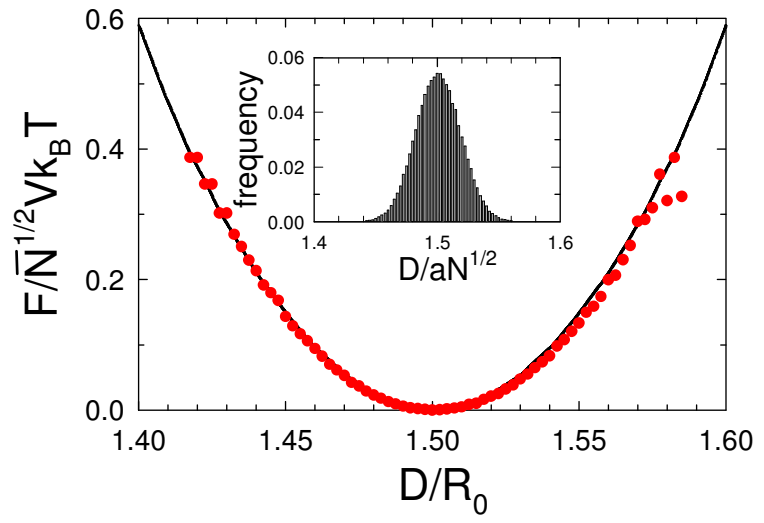

FIG. 4. Free energy density of LAM, varying domain spacing, $D$, at $\chi_{b} N=16$, for a system containing 2 periods. Free energies are given relative to the minimum. The black line was calculated using thermodynamic integration and the red points were calculated using the box move. The thermodynamic integrals were done using $1.5 \times 10^{-6} \mathrm{D} /$ step and the box move data were evaluated using $10^{6}$ steps. The inset shows a histogram of the data obtained from the box move, which is used to find the free energy. ${ }^{1}$

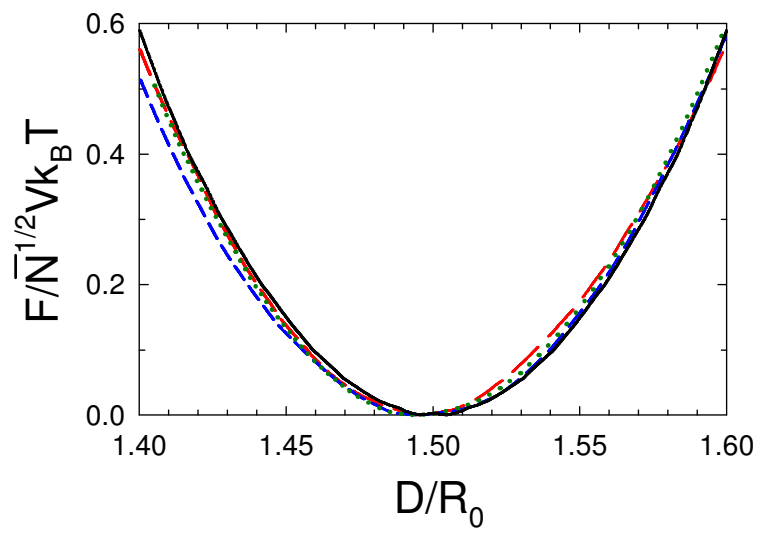

FIG. 5. Free energy densities of LAM, varying domain spacing, $D$, calculated using thermodynamic integration. Curves correspond to cubic systems containing 1 (black, solid), 2 (red, dashed), 3 (blue, dashed) and 4 (green, dotted) lamellae at $\chi_{b} N=16$. Integrals were done using steps of $1.5 \times 10^{-6} \mathrm{D} / \mathrm{step}$.

effects. The resulting data are shown in Figure 5. The free energies show almost no dependence on system size, suggesting that finite-size effects are small.

For a thorough examination of finite-size effects, we also calculate the structure function for DIS and LAM, illustrated in Figure 6 . The structure function, $S(\mathbf{k})$, is a function of the wave vector, $\mathbf{k}$. It is also convenient to average $S(\mathbf{k})$ over a sphere of radius $k$ to obtain the angleaveraged structure function, $S(k)$. In DIS, the values of $S(k)$ lie on the same curve, however, as $h$ decreases, the number of points decreases and the small wave vector cutoff decreases. In the lamellar phase, $S(\mathbf{k})$ is dominated 

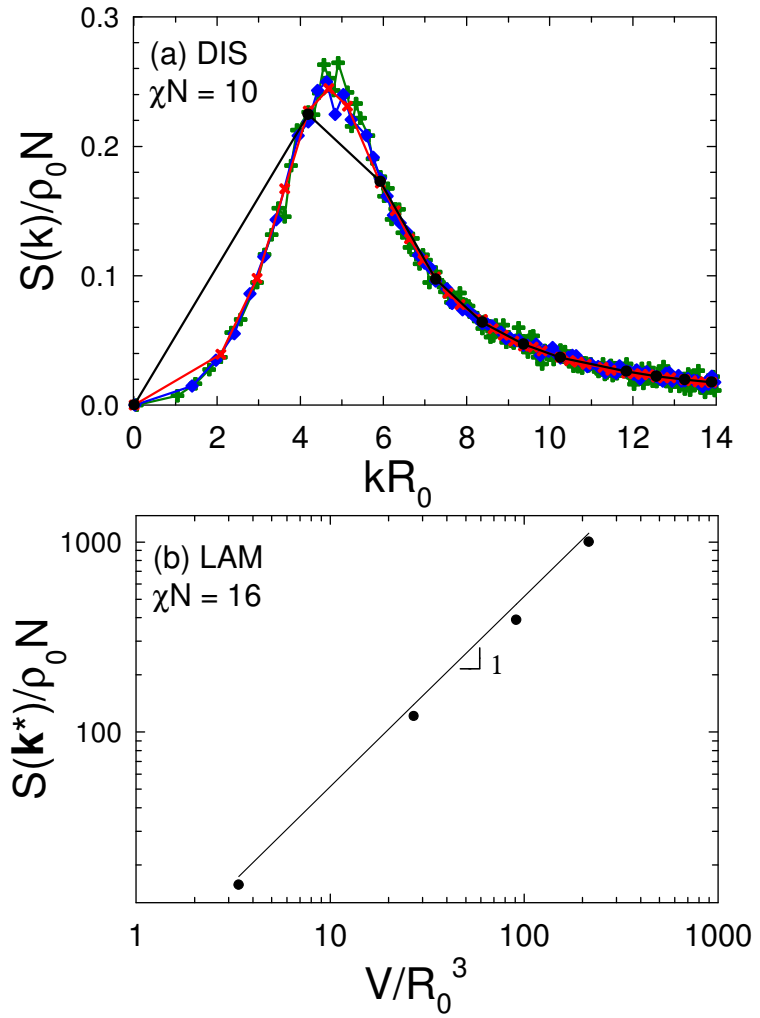

FIG. 6. Structure function data for (a) DIS and (b) LAM. For DIS, the angle average structure function is calculated at $\chi_{b} N=10$. Simulation boxes are cubic, with a side length corresponding to 1 (black circles), 2 (red $\times \mathrm{s}$ ), 3 (blue diamonds) and 4 (green $+\mathrm{s}$ ) lamellar periods. For LAM, the hight of the peak in the structure function at the principle wave vector, $\mathbf{k}^{*}$, is shown for $\chi_{b} N=16$. A line with slope 1 is shown to illustrate how $S\left(\mathbf{k}^{*}\right)$ scales with $V$.

by a peak at $\mathbf{k}^{*}$, which shows the expected scaling, ${ }^{10,11}$ even down to $h=1$.

Angle-averaged structure functions, such as in Figure 6(a), are evaluated by finding $S(\mathbf{k})$, and averaging over grid points with the same $k$. This works well for direction-independent fluctuations, such as in the disordered phase. Direction-dependent fluctuations, however, can present difficulty. To see why, consider that the structure function at the dominant wave vector in the lamellar phase is dominated by $S\left( \pm \mathbf{k}^{*}\right) \propto V$. A (discrete) angleaveraged becomes $2 S\left(\mathbf{k}^{*}\right) / l$, where $l$ is the number of grid points intersecting a sphere of radius $k^{*}$. $l$ depends on the geometry of the grid and for our cubic latices with $h=1,2,3$ and 4 , the numbers of points on spheres of radius $k^{*}$ are $6,6,30$ and 6 respectively. This leads to apparently strange behavior in $2 S\left( \pm \mathbf{k}^{*}\right) / l$ for $h=3$. The reader is cautioned to consider this when examining angle-averaged structure functions of ordered phases represented on discrete grids.

\section{DISCUSSION}

When calculating the ODT, we used $h=2,3$ and 4 lamellar periods, whereas when investigating the structure function and compression of lamellae, we also included $h=1$. Close to the ODT, the melt may spontaneously order or disorder. This occurs more for small than large systems. The difference may be because the length scale of lamellar or disordered fluctuations is closer to the system size, causing the system to be more easily overtaken thereby. This is known to happen in simulations with periodic boundary conditions when the critical nucleus size is close to the system size. ${ }^{24}$ It may also be because the relaxation time of the system corresponds to a smaller number of simulation steps. These phase changes lead to difficulty calculating $F$ vs. $\chi_{b} N$ and thus in locating the ODT. We therefore did not include $h=1$ data when locating the ODT. At higher $\chi_{b}$, where we calculated $F$ vs. $D$ and $S(k)$, the simulations did not have this tendency to change phase and data were obtainable.

The ease of transitioning between DIS and LAM, which creates the above difficulty, arises because the ODT for symmetric copolymers is only weakly first-order. This is apparent from the small difference between the free energy slopes (which gives the latent heat) in DIS and LAM in Figure 2. As $\bar{N}$ increases, the latent heat decreases further and as $\bar{N} \rightarrow \infty$ the transition becomes continuous, rendering thermodynamic integration unable to find the ODT. We tried to repeat our procedure for $\bar{N}=10^{6}$ but were unsuccessful, as the difference in slopes of $F$ vs. $\chi_{b} N$ and free energies between DIS and LAM became so small that we could not confidently identify the ODT within a reasonable uncertainty. This difficulty in calculating the ODT for symmetric diblock copolymers at $\bar{N}=10^{6}$ may help explain why Lennon et al. needed to extrapolate their free energy curves, as well as explaining the difference between their ODT and other determinations. ${ }^{2} \mathrm{We}$ expect these problems to diminish for more strongly firstorder transitions, including many order-order transitions as well as the ODT further from $f=1 / 2$.

The weakly first-order nature of the transition makes the ODT at $f=1 / 2$ the most difficult transition to consider using thermodynamic integration. The opposite is the case for metastability interval method, ${ }^{1}$ as the ease of transitioning between DIS and LAM leads to tight bracketing of the ODT. The fact that continuous thermodynamic integration was able to achieve superior precision highlights the utility of this method.

Our difficulty in locating an ODT for $\bar{N}=10^{6}$, as well as the large error bars for $h=4$, emphasizes the necessity for taking care when performing this type of calculation. If uncertainties are not carefully considered, it is easy to locate a seemingly correct, but fallacious ODT. This was particularly apparent when we redid the calculation in Figure 2(a) using 8 times fewer steps and approximately tripled the range given by the bounds on the ODT.

One seemingly odd result is that on increasing the system size, the free energy density of the melt increases. 
We would expect that increasing the number of excitable modes would increase the entropy and thus decrease the free energy. One possible explanation comes from distinguishability in the EC reference state. The reference free energy density, that of the EC, is assumed to be the same for each system. This is only true if the oscillators are distinguishable leading to an extensive free energy. Modifying the free energy to assume the oscillators are indistinguishable, however, overestimates the effect and produces a significant decrease in free energy with increasing system size. Although we do not know the source of the behavior, we note that other work has found finite-size corrections of the same sign using thermodynamic integration from an Einstein crystal reference state. $^{25}$

In the lamellar phase, the $W_{0}(\mathbf{r})$ reference field holds the lamellae at one particular position. In the $\lambda \rightarrow 1$ limit, the system therefore gains the ability to drift and thus gains translational entropy (Goldstone mode excitation). This single additional degree of freedom should provide a contribution to the total free energy of order $k_{B} T$. Its influence on the free energy per unit volume is minuscule, particularly for large systems. Nonetheless, Delaney and Fredrickson noticed a contribution to free energy derivatives that appeared to be from this drift. ${ }^{5}$ It entered through the $H_{\mathrm{EC}}$ term in the derivative (see Equation (12)) and depended on both system size and length of the simulation. They excluded the $\lambda \rightarrow 1$ limit by using open Newton-Cotes quadrature for their integrals, thereby obtaining free energy densities which did not depend on system size or simulation duration. We do not see the aforementioned drift because the system does not spend a sufficient number of steps at or near $\lambda=1$ to drift.

The statistical uncertainty in our calculation is given by the difference between the forward and back integrals. This uncertainty can be decreased in a number of ways. One obvious way is to use more Monte Carlo (MC) steps. Another way is to conduct many thermodynamic integrals and use the Jarzynski relation, which relates averages over these integrals to the true free energy difference. ${ }^{22}$ This procedure is computationally intensive and it is unclear if additional computational resources would be better utilized by simply increasing the number of MC steps. Another option, if the systematic error varies significantly with $X$, is integrating more slowly over the problematic intervals. The integration rate could be adjusted automatically during a simulation by periodically integrating back a short distance, to determine the relative size of the systematic error, and adjusting appropriately.

When describing continuous thermodynamic integrals, we indicated that $d H / d X$ was calculated and the free energy updated every MC step. Subsequent steps in a MC simulation are often quite correlated, making many of these data superfluous. It is more efficient to collect data less often, say every $m$ steps. If data are not being collected during these steps, it may be advantageous to only update the integration variable, $X$, every $m$ steps, allowing the system to equilibrate to the new $X$ before $d H / d X$ is calculated. We tested this modification in the $\lambda$ integral, using $m=10$ and $h=4$, and found a $10 \%$ reduction in the uncertainty in the $\mathrm{BCP}$ free energy for both phases. The increased precision at no extra computational cost suggests that this modification is worth exploring further. We suspect that increasing $m$ further would lead to more precise results, however, too large an $m$ would lead to random and finite-step errors.

\section{SUMMARY}

Continuous thermodynamic integration (TI) has provided us with a fast, precise way of examining a block copolymer melt using Monte Carlo field-theoretic simulations. We investigated the location of the ODT, equilibrium lamellar spacing and compression modulus for various system sizes and found no evidence for finite-size effects. Perhaps this should not be too surprising, since the structure function shows us that long wavelength contributions to the structure are small. The uncertainty in the ODT increases quickly with system size, meaning that one should be more concerned about the precision of results when simulating larger systems. This is particularly concerning when the errors are not carefully considered. The lack of apparent finite-size effects combined with the large uncertainties found for large systems suggest that small volumes may be preferable in simulations.

The ODT and compression modulus can, and have, been found by other means, yielding results that are consistent with ours. ${ }^{1}$ Continuous TI, however, allows for more precise results, obtained faster than traditional TI or the other methods discussed. It also offers a more straightforward error calculation than traditional TI. We calculated the stretching and compression energy of lamellae an order of magnitude faster with TI than with the box move, and we can find the ODT at system sizes that take prohibitively long for the metastability interval method. The increase in speed and precision will likely continue to improve for order-order transitions and asymmetric copolymers.

\section{ACKNOWLEDGMENTS}

This work was funded through a collaboration with the University of Minnesotas Center for Sustainable Polymers (CHE-1413862). Calculations were conducted using the facilities of the Shared Hierarchical Academic Research Computing Network (SHARCNET) and Compute Canada. 
*r6spence@uwaterloo.ca

1 Vorselaars, B.; Stasiak, P.; Matsen, M. W. Macromolecules 2015, 48, 9071-9080.

${ }^{2}$ Lennon, E. M.; Katsov, K.; Fredrickson, G. H. Phys. Rev. Lett. 2008, 101(13), 138302.

${ }^{3}$ Frenkel, D.; Ladd, A. J. C. J. Chem. Phys. 1984, 81(7), 3188.

4 Müller, M.; Daoulas, K. C. J. Chem. Phys. 2008, 128(2), 024903.

${ }^{5}$ Delaney, K. T.; Fredrickson, G. H. J. Phys. Chem. B 2016, 120(31), 7615-7634.

6 Watanabe, M.; Reinhardt, W. P. Phys. Rev. Lett. 1990, 65(26), 3301-3304.

7 Zacharias, M.; Straatsma, T. P. J. Chem. Phys. 1994, page 9025.

8 Tuckerman, M. Statistical mechanics: theory and molecular simulation; Oxford University Press, New York, USA 2010.

${ }^{9}$ Vorselaars, B. J. Chem. Phys. 2015, 142(11), 114115.

10 Micka, U.; Binder, K. Macromol. Theory Simul. 1995, 4(3), 419-447.

11 Arora, A.; Morse, D. C.; Bates, F. S.; Dorfman, K. D. Soft Matter 2015, 11(24), 4862-4867.

12 M. W. Matsen in Soft Matter, Volume 1: Polymer Melts and Mixtures (Eds: G. Gompper, M. Schick), Wiley-VCH, Weinheim, Germany 2006

13 Müller, M.; Schmid, F. In Advanced Computer Simulation Approaches for Soft Matter Sciences II; Holm, C., Kremer, K., Eds.; Springer-Verlag: Berlin, Germany, 2005.

14 Fredrickson, G. The Equilibrium Theory of Inhomogeneous Polymers (International Series of Monographs on Physics); Oxford University Press, Oxford, UK, 2006.

15 Stasiak, P.; Matsen, M. W. Euro. Phys. J. E, Soft matter 2011, 34(10), 110.

16 Spencer, R. K. W.; Matsen, M. W. Macromolecules 2016, 49(16), 6116-6125.

17 Olvera de la Cruz, M.; Edwards, S. F.; Sanchez, I. C. J. Chem. Phys. 1988, 89(3), 1704-1708.

18 Düchs, D.; Ganesan, V.; Fredrickson, G. H.; Schmid, F. Macromolecules 2003, 36(24), 9237-9248.

19 Stasiak, P.; Matsen, M. W. Macromolecules 2013, 46(19), 8037-8045.

20 Matsen, M. W. Euro. Phys. J. E, Soft matter 2009, 30(4), 361-369.

21 Fredrickson, G. H.; Helfand, E. J. Chem. Phys. 1987, 87(1), 697-705.

22 Jarzynski, C. Physical Review Letters 1997, 78(14), 26902693.

23 Riggleman, R. A.; Fredrickson, G. H. J. Chem. Phys. 2010, 132(2), 024104-13.

24 Honeycutt, J. D.; Andersen, H. C. Chemical Physics Letters 1984, 108(6), 535-538.

25 Polson, J. M.; Trizac, E.; Pronk, S.; Frenkel, D. J. Chem. Phys. 2000, 112(12), 5339. 
Table of Contents description:

Continuous thermodynamic integration is used to determine equilibrium behavior in copolymer melts, with speed and accuracy that is unprecedented in fieldtheoretic simulations. Integrating from an Einstein crystal reference state yields absolute free energies, making our method extensible to order-order transitions. Equilibrium properties show negligible finite-size effects, however, uncertainties therein increase with system size, perhaps making small systems preferable for simulations.

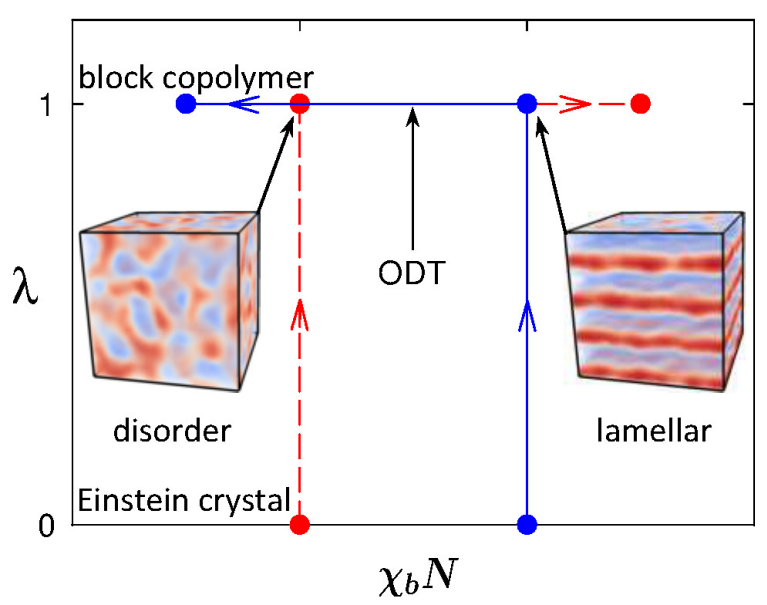

FIG. 7. Table of contents and abstract figure. 